\title{
Konstruktionen von Männlichkeit in der Antike und der paulinischen Korintherkorrespondenz*
}

\author{
Moisés Mayordomo
}

\begin{abstract}
Männlichkeit bildet in der griechisch-römischen Antike eine zentrale Kategorie zur Strukturierung sozialer Wirklichkeit. Dabei muss Männlichkeit durch öffentliche Selbstdarstellung in Form von Kontrolle und Herrschaft erworben und erhalten werden. Gegenüber diesem Konzept von Männlichkeit setzt Paulus insbesondere in der Korintherkorrespondenz eigene Akzente, ohne dabei mit den herrschenden Deutungsvorgaben völlig zu brechen. Einerseits entzieht er sich den Konkurrenzmechanismen öffentlicher Rede und den Erwartungen männlicher Härte. Andererseits verhält er sich in seiner Ablehnung langhaariger Männer und in seinem Festhalten an der binären Struktur symbolischer Geschlechterdarstellung völlig normkonform. Er stellt die aktive Rolle des Mannes nicht in Frage, qualifiziert diese allerdings durch die Beziehung zu Christus und durch das Verständnis von Liebe als Autonomieverzicht. Mit Paulus bahnt sich eine wichtige Entwicklung an, die sexuelle Enthaltsamkeit und Selbstkontrolle als Form männlicher Kontrolle deutet.
\end{abstract}

\section{Ein Blick in die Matrix der Männlichkeit}

»Du kennst die geschniegelten jungen Männer, Bart und Haupthaar glänzend, ganz aus dem Schmuckkästchen: nichts Mannhaftes (nibil forte) kannst du von ihnen erhoffen, nichts Gediegenes (nihil solidum). Die Sprache ist das Kleid der Seele; wenn sie wohlfrisiert, geschminkt und künstlich hergerichtet ist, zeigt sie, daß auch die Seele ihrer nicht sicher ist und etwas Gebrochenes an sich hat. Nicht ist sie ein Mannesschmuck (ornamentum virile) - gedrechselte Ausdrucksweise. Wenn wir in das Herz eines Mannes von Wert (animum boni viri) blicken könnten - o, welch schönen Anblick, welch ehrwürdigen, von Größe und Ausgeglichenheit leuchtenden sähen wir, hier strahlte Gerechtigkeit, dort Tapferkeit, dort Mäßigung und Klugheit. [...] Wenn jemand diese Gestalt erblickt, höher und leuchtender, als man es unter Menschen zu sehen bekommt, bliebe er nicht wie bei der Begegnung mit einer Gottheit angewurzelt stehen und bäte stumm, es möge ihm gestattet sein, das zu sehen? Wenn der gütige Ausdruck des Antlitzes selbst dazu aufforderte, träte er näher und betete andächtig und kniefällig zu ihr. « ${ }^{1}$

* Es handelt sich um den geringfügig veränderten Text eines März 2006 an der Universität Bern gehaltenen Vortrags. Für kritische Rückfragen und hilfreiche Vorschläge danke ich Prof. Wolfgang Lienemann, Prof. Ulrich Luz, Julia Müller-Clemm, Christine Rosin und den kritischen Geistern des Netzwerks Geschlechterbewusste Theologie (v. a. Heike Walz, Tania Oldenhage, Martin Fischer und Ruth Hess).

1. Seneca, Ep. mor. 115,2-4 (übers. Rosenbach).

Evang. Theol. 68. Jg., Heft 2, S. 99-115

ISSN 0014-3502 @ Chr. Kaiser / Gütersloher Verlagshaus, 2008 
Die Vergöttlichung des römischen Mannes - nicht mehr und nicht weniger - unternimmt hier Seneca, ein Zeitgenosse des Apostels Paulus. Eine derart vollendete Symbiose von moralischer Tugend, körperlicher Ausstrahlung und rhetorischer Leistung war kaum an der Tagesordnung. Dennoch entstammt eine solche Idealprojektion nicht der zufälligen Phantasie eines Einzelnen. Sie erhält vielmehr ihre Bedeutung innerhalb einer sozialen Matrix, in der die Kategorie des Männlichen in sämtliche Lebensbereiche hineinragt. ${ }^{2}$ Der antike Männlichkeitsdiskurs ist an unzähligen Stellen ablesbar: in juristischen, rhetorischen, physiognomischen und medizinischen Lehrbüchern, bei Historikern, Dichtern, Satirikern und Traumdeutern, auf den Bühnen der Dramen und Komödien, in repräsentativen Statuen und Münzen und an vielen Wänden mit ihren bildlichen Darstellungen und unzweideutigen Graffiti. Die hellenistischen Moralphilosophen aller Richtungen diskutieren die zentrale Bezie-

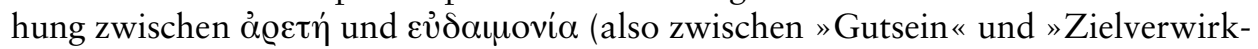
lichung «) meist im Kontext der Handlungsoptionen des freien, erwachsenen Mannes. ${ }^{3}$ Unter der strengen Leitung des pater familias, des Hüters und Überlieferers männlicher Werte, durchliefen die Jungen in der Adoleszenz eine schwere »Schule der Männlichkeit « ${ }^{4}$. Die bevorzugten Orte für den Vollzug von Männlichkeit waren - um nur die wichtigsten zu nennen - Krieg und Militär, ${ }^{5}$ politische Ämter und öffentliche Rede (II.2.), sportlicher Wettkampf ${ }^{6}$ und der verzweigte Bereich von Ehe, Familie und Sexualität.

Es ist diese durchgehende Präsenz des Männlichen, die seine materielle Abgrenzung und methodische Analyse ungemein erschwert. Aus diesem kaum überschaubaren Netz von Verstrickungen, Bündnissen und Kooperationen kann im Folgenden nur ein Ausschnitt beleuchtet werden. Der spezifische Fokus ist davon abhängig, in

2. Vgl. zum antiken Männlichkeitsdiskurs L. Foxhall/J. Salmon (Hg.), Thinking Men. Masculinity and its Self-Representation in the Classical Tradition, London/New York 1998; dies. (Hg.), When Men Were Men. Masculinity, Power and Identity in Classical Antiquity, London/New York 1998; M. W. Gleason, Making Men. Sophists and Self-Presentation in Ancient Rome, Princeton 1995; M. Golden/P. Toohey (Hg.), Sex and Difference in Ancient Greece and Rome, Edinburgh 2003; E. Gunderson, Staging Masculinity. The Rhetoric of Performance in the Roman World, Ann Arbor 2000; H. Moxnes, Conventional Values in the Hellenistic World. Masculinity, in: P. Bilde u. a. (Hg.), Conventional Values of the Hellenistic Greeks (Studies in Hellenistic Civilization 8), Aarhus 1997, 263-284; R. M. Rosen/I. Sluiter (Hg.), Andreia. Studies in Manliness and Courage in Classical Antiquity (Mn.S 238), Leiden/Boston 2003; T. Späth, Männlichkeit und Weiblichkeit bei Tacitus. Zur Konstruktion der Geschlechter in der römischen Kaiserzeit, Frankfurt a. M./New York 1994; M. Wyke (Hg.), Parchments of Gender. Deciphering the Bodies of Antiquity, Oxford 1998.

3. Im Falle der Tapferkeit ( $\alpha v \delta \varrho \varepsilon i ́ \alpha)$ ist der Bezug zur Mannhaftigkeit sprachlich ebenso naheliegend wie beabsichtigt (vgl. K. Bassi, The Semantics of Manliness in Ancient Greece, in: Rosen/Sluiter, a.a.O., [s. Anm. 2], 25-58 und M. McDonnell, Roman Men and Greek Virtue, a.a. O., 235262). Im lateinischen ist dieser Nexus dadurch sprachlich festgeschrieben, dass die Römer das

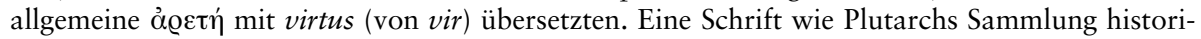

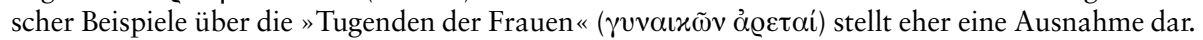
Vgl. zur aristotelischen Konzeption M. Deslauriers, The Virtues of Women and Slaves, in: Oxford Studies in Ancient Philosophy 25 (2003), 213-231.

4. Vgl. C. Humpert, Wege zur Männlichkeit im Rom der Späten Republik. Cicero und die adulescentia seiner Zeit, Halle 2001 (S. 8: "Schule der Männlichkeit«); Späth, a.a.O., (s. Anm. 2), 290306.

5. Vgl. R. Alston, Arms and the Man. Soldiers, Masculinity and Power in Republican and Imperial Rome, in: Foxhall/Salmon, When Men Were Men (s. Anm. 2), 205-223.

6. O. v. Nijf, Athletics, Andreia and the Askesis-Culture in the Roman East, in: Rosen/Sluiter, a. a. O. (s. Anm. 2), 263-286. 
welcher Hinsicht die neutestamentlichen Texte befragt werden sollen. Im Vergleich zu den klassisch-philologischen Nachbardisziplinen steht die diesbezügliche neutestamentliche Forschung in den Anfängen. ${ }^{7}$ In einem der rar gesäten deutschsprachigen Beiträge zur Thematik umreißt Martin Leutzsch vier mögliche Fragestellungen: Die narrative Charakterisierung von männlichen Figuren in erzählenden Prosatexten, die »Konstruktionen der Männlichkeit Jesu in den Evangelien «, die männliche Konzeptualisierung von Gott, und die historische Frage nach den frühchristlichen »Konstruktionen von Männlichkeit « in Texten, »in denen Normen zu Männlichkeit artikuliert, begründet und entfaltet werden ${ }^{8}$.

Die Korintherkorrespondenz bietet ein geeignetes Explorationsfeld zu der zuletzt genannten Frage. Im Rahmen des antiken Männlichkeitsdiskurses erhält die komplexe Interaktion zwischen Paulus und den Korinthern neue Konturen. Im Hinblick auf das spezifisch römische Kolorit der Stadt Korinth und die in beiden Briefen behandelten Themen sollen im Folgenden Aspekte aus dem Bereich Körperlichkeit, Herrschaft und Sexualität im Mittelpunkt stehen.

\section{Der römische Mann - Körper in und unter Kontrolle}

\section{Der Körper der Medizin und seine mythologische Verarbeitung}

Die Unterscheidung zwischen biologischem und sozialem Geschlecht (engl. sex und gender) gehört zu den zentralen Kategorien im aktuellen Geschlechterdiskurs. Obwohl gerade das biologische Geschlecht häufig als kulturübergreifende Naturgegebenheit betrachtet wird, zeigt ein Blick in die Medizingeschichte, dass auch die Wahrnehmung und Konzeptualisierung biologischer Unterschiede historischen Wandlungsprozessen unterliegt. ${ }^{9}$ Selbstverständlich galten auch in der Antike die äußeren Sexualorgane als primäre geschlechtliche Attributionsmerkmale. ${ }^{10}$ Das Verhältnis des weiblichen Körpers zum männlichen wurde jedoch anders konzeptionalisiert. Im 2. Jh. n. Chr. bringt der einflussreiche Arzt und Philosoph Galenos die Schulmeinung auf den Punkt:

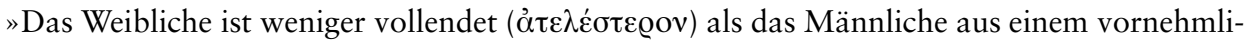
chen Grund, weil es kälter ( $\psi v \chi \varrho o ́ \tau \varepsilon \varrho o v)$ ist. Wenn nämlich unter den Tieren das Wärmere das Aktivere ist, so wäre das kältere (Tier) weniger vollendet als das wärmere. Ein zweiter Grund

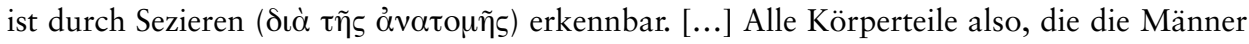

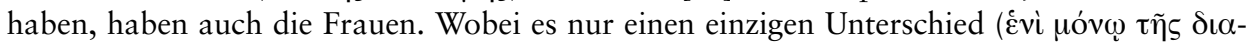

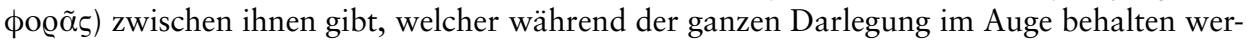
den muss: Die Körperteile der Frauen sind innen ( $\left.\varepsilon^{\prime} v \delta o v\right)$, während die des Mannes außen $\left({ }^{\prime} \xi \omega\right)$ sind [...]. Denke nun zunächst nach Belieben an irgendwelche der jeweiligen Körpertei-

7. Vgl. die Aufsätze in S. D. Moore/J. C. Anderson, New Testament Masculinities (Semeia Studies 45), Atlanta 2003.

8. M. Leutzsch, Konstruktionen von Männlichkeit im Urchristentum, in: F. Crüsemann u. a. (Hg.), Dem Tod nicht glauben. Sozialgeschichte der Bibel (FS L. Schottroff), Gütersloh 2004, 601.

9. Grundlegend zur Geschichte des Körpers T. Laqueur, Auf den Leib geschrieben. Die Inszenierung der Geschlechter von der Antike bis Freud, München 1996.

10. Insbesondere im römischen Recht war es wichtig, den legalen Status des Kindes von Geburt an zu bestimmen (vgl. J. F. Gardner, Sexing a Roman. Imperfect Men in Roman Law, in: Foxhall/Salmon, When Men Were Men [s. Anm. 2], 136-152). 


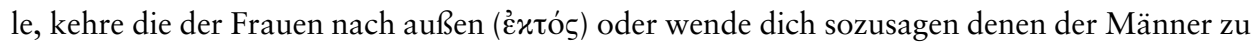
und falte sie doppelt nach innen ( $\left.\varepsilon^{\prime} \sigma \omega\right)$ und du wirst das im Verhältnis zueinander völlig Glei-

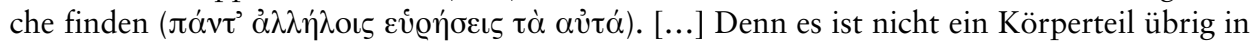
den Männern zu finden, außer dass es den Platz vertauscht hat, denn die (Körperteile, die) bei

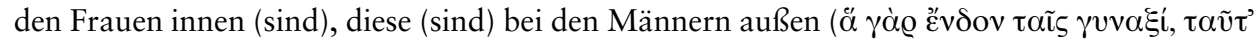

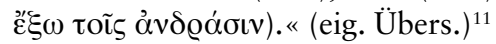

Gemäß dieser Konzeption ist der männliche Körper immer auch weiblich - und umgekehrt. Entsprechend wurde in der antiken Zeugungslehre die Auffassung vertreten, dass Mann und Frau über männlichen und weiblichen Samen verfügen. Das jeweilige Mischungsverhältnis bestimmt nicht nur, ob ein Junge oder ein Mädchen geboren wird, sondern entscheidet im Hinblick auf beide über den Grad an Männlichkeit bzw. Weiblichkeit. ${ }^{12}$

Diese medizinischen Objektivierungen wurden in der Antike durch Mythen untermauert, die von der Vorstellung eines eingeschlechtlichen Körpers zeugen. ${ }^{13}$ Die Angst vor dem Verlust sexueller Identität spiegelt sich in Legenden wider, die von einem spontanen Geschlechtswechsel berichten. Hier zwei kurze Beispiele aus der Wundersammlung des Phlegon von Tralleis (2. Jh. n. Chr.):

»Ein Mädchen namens Philotis, das aus Smyrna stammte, war im heiratsfähigen Alter und von ihren Eltern mit einem Mann verlobt, als männliche Genitalien an ihr erschienen und sie zu einem Mann wurde. Es gab einen weiteren Androgynen zu eben jener Zeit [53 n. Chr.] in Epidauros, ein Kind armer Eltern, das früher Sympherousa geheißen hatte, aber nachdem es zu einem Mann geworden war, Sympheron hieß. Er verbrachte sein Leben als Gärtner. « ${ }^{14}$

Da das Weibliche im männlichen Körper präsent ist - und umgekehrt -, ist der Körper der Ort, an dem die Polarität zwischen den Geschlechtern ausgetragen wird. ${ }^{15}$ Angesichts der stets inhärenten »Gefahr« (aus damaliger Sicht) der Verweiblichung

11. Galenos, De usu partium 14,6-7 (ed. Kühn, IV, 159f.); vgl. De semine 2,1f.5 (ed. Kühn IV 596f.634-636).

12. Vgl. Corpus Hippocraticum (5.-4. Jh. v. Chr.), De genitura (Über die Zeugung) 6-7 (in: C. Schubert/U. Huttner, Frauenmedizin in der Antike [TuscBü], Düsseldorf 1999, 108-111 [dort S. $514 \mathrm{f}$. zur sog. Pangenesis-These]); De victu (Über die Diät) 28 f. (= Schubert/Huttner, a. a. O., 118-121); Galenos, De usu partium 14,7 (ed. Kühn, IV, 171f.); Gleason, a.a.O. (s. Anm. 2), 59 und die Artikel in K.-H. Leven (Hg.), Antike Medizin. Ein Lexikon, München 2005 von K.-H. Leven, »Embryo « (Sp. 249-251) und von S. Föllinger, »Geschlecht « (Sp. 339-342) und »Samen « (Sp. 763 f.). Die spätere medizinische Tradition tendierte dazu, den Beitrag der Frau bei der Zeugung möglichst gering zu halten (vgl. M. Harlow, In the Name of the Father. Procreation, Paternity and Patriarchy, in: Foxhall/Salmon, Thinking Men (s. Anm. 2), 155-169; K. Aspegren, The Male Woman. A Feminine Ideal in the Early Church [AUU A.4], Stockholm 1990, 16-19.34-36.54f.).

13. Vgl. zum Mythos eines ursprünglichen androgynen Urmenschen Plato, Symp. 189d-191d. Er findet sich auch in der orthodoxen rabbinischen Deutung der Schöpfungserzählung im palästinensischen Midrash Genesis Rabbah (ca. 5. Jh. n. Chr.) zu Gen 1,26 (A. Wünsche, Der Midrasch Bereschit Rabba, Leipzig 1881, 29f.). Vgl. dazu D. Boyarin, Carnal Israel. Reading Sex in Talmudic Culture, Berkeley 1993, 35-46; W. A. Meeks, The Image of the Androgyne. Some Uses of a Symbol in Earliest Christianity, in: HR 13 (1973/1974), 184-189.

14. Buch der Wunder 7,2; 8 (П飞@i $\theta \alpha v \mu \alpha \sigma i \omega v$, hg. u. übers. K. Brodersen, 48 f.). Die Verbreitung und Popularität solcher Sensationsliteratur belegt Aulus Gellius (etwa 130-180), Noctes Atticae 9,4, der in einem Buchmarkt in Brundisium zahlreiche griechische Werke mit Wundererzählungen von namhaften Autoren vorfindet. Vgl. weiterhin Plinius d. Ä., Nat. Hist. 7,34.36.

15. J. J. Winkler, Der gefesselte Eros. Sexualität und Geschlechterverhältnis im antiken Griechenland, Marburg 1994, 80. 
ist Männlichkeit kein Gegebenes, sondern etwas, das erkämpft und erhalten werden muss. ${ }^{16}$

\section{Der Körper des Redners und der Traum der Physiognomisten}

Wie in vielen anderen Kulturen konstituierte sich Maskulinität auch in der römischen Antike primär im Modus der öffentlichen Selbstrepräsentation. Sie stand immer auf dem Prüfstand, wurde stets verhandelt und änderte ihren »Wert « mit dem Verlust oder mit der Vermehrung persönlicher Ehre. ${ }^{17}$ Die öffentliche Rede galt als eines der wichtigsten kompetitiven Felder für den Erweis von Männlichkeit. ${ }^{18}$

Die Aufmerksamkeit der Zuhörer wie der Experten galt nicht nur dem Inhalt des Gesagten, sondern auch Aspekten der körperlichen Erscheinung, Gestik, Mimik und Stimme. Vor dem Hintergrund der physiognomischen Überzeugung, dass Seele und Körper in Wechselwirkung miteinander stehen ${ }^{19}$, gilt der Körper als übercodierter Referenzträger für den Charakter eines Mannes. ${ }^{20}$ Der Körper verrät nicht nur, wer feige und träge ist, er gibt auch den Grad an Männlichkeit preis. ${ }^{21}$ Der in die Öffentlichkeit tretende männliche Körper war daher bis ins Detail kartographiert: Jede Gesichtsregung, Handbewegung oder Modulation der Stimme, jede körperliche Abweichung und anatomische Zufälligkeit unterlag einer strengen Überwachung. ${ }^{22} \mathrm{Ab}-$ weichungen vom Standard konnten nach damaliger Sicht einen Mann in gefährliche Nähe zum Weiblichen rücken und damit seine Autorität als Redner untergraben. Es

16. Gleason, a.a. O. (s. Anm. 2), 159. Das weite Gebiet von "männlichen Frauen « kann hier nicht betrachtet werden (vgl. Aspegren, a.a. O. [s. Anm. 12]; J. McInerney, Plutarch's Manly Women, in: Rosen/Sluiter, a.a. O. [s. Anm. 2], 319-344).

17. Vgl. die anthropologisch vergleichende Untersuchung von D. D. Gilmore, Mythos Mann. Rollen, Rituale, Leitbilder, München/Zürich 1991.

18. Ausführlich dargelegt in Gleason, a. a. O. (s. Anm. 2); s. a. Gunderson, a. a. O. (s. Anm. 2); Ders., Discovering the Body in Roman Oratory, in: Wyke, a.a. O. (s. Anm. 2), 169-189; A. Richlin, Gender and Rhetoric. Producing Manhood in the Schools, in: W. J. Dominik (Hg.), Roman Eloquence. Rhetoric in Society and Literature, London/New York 1997, 90-110.

19. (Ps.-)Aristosteles, Physiog. A 1,805a1-12; B 4,808b11-14.

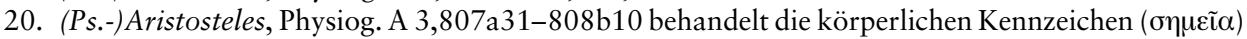
des Mutigen, Feigen, Begabten, Stumpfsinnigen, Unverschämten, Anständigen, Heiteren, Traurigen, Kinäden, Verbitterten, Ungestümen, Sanftmütigen, Ironikers, Kleinmütigen, Würfelspielers, Tänzers, Schmähsüchtigen, Mitleidigen, Essers, Lüsternen, Schlafliebenden, Schwatzhaften, Erinnerungsfähigen. Die logische Grundlegung der Physiognomik findet sich bei Aristoteles in An pr. II 27.

21. Vgl. zur Physiognomik »weibischer « Männer E. Meyer-Zwiffelhoffer, Im Zeichen des Phallus. Die Ordnung des Geschlechtslebens im antiken Rom (Historische Studien 15), Frankfurt a. M. 1995, 139-153. Als Beispiel Diog L., 7,173 (übers. O. Apelt): »Auch folgendes Geschichtchen wird von ihm [sc. Kleanthes] erzählt: Als er einmal im Sinne Zenons die Bemerkung machte, man könne den Charakter schon aus dem Äußeren erkennen, hätten einige zu Späßen aufgelegte Jünglinge einen Wollüstling (xivoıঠov), der auf dem Lande in harter Zucht hatte leben müssen, zu ihm gebracht mit der Aufforderung, über seinen Charakter Auskunft zu geben; er hätte nach einigem Schwanken den Menschen weggehen heißen. Beim Weggehen nieste er. Da sagte Kleanthes: >Da habe ich

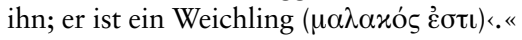

22. Quintilian widmet einen ausführlichen Abschnitt seines rhetorischen Lehrbuchs den Themen Gestik und Kleidung (Inst. XI 3,61-149 = Rahn, II, 632-665). Die Abgrenzung von allem Weiblichen ist im Vortrag wichtig (vgl. XI 3,32.128 und die Darstellung in Gunderson, a.a. O. [s. Anm. 2], 59-110). Vgl. aus der römischen rhetorischen Tradition Seneca, Ep. mor. 114; Cicero, Orator 18,59 . 
ist kaum auszudenken, wie viele Fehler ein nicht perfekt geschulter Redner vor einem römischen Publikum unwissentlich begehen konnte!

Die Physiognomisten, die den Körper »wissenschaftlich « deuteten und normierten, waren ganz besonders um die Abgrenzung zwischen männlichen und weiblichen Körpern und den damit einhergehenden Charaktereigenschaften bemüht. ${ }^{23}$ Der römische Redner und Physiognomist Polemon aus Laodizäa gibt im 2. Jh. n. Chr. die folgende Empfehlung:

»Du kannst physiognomische Hinweise für Männlichkeit und Weiblichkeit (signis masculini et feminini) erhalten durch die Sehkraft, die Bewegung und die Stimme deines Gegenübers. Vergleiche aus diesen Zeichen die einzelnen miteinander, bis du zu deiner Zufriedenheit feststellen kannst, welches der beiden Geschlechter dominiert. Denn im Männlichen ist Weibliches und im Weiblichen Männliches (nam in masculino femininum et in feminino masculinum est), aber der Begriff ('männlich` und 'weiblich`) wird zugewiesen, je nachdem welches von beidem überwiegt. «(Physiogn. 2 [25r]; Übers. nach Gleason) ${ }^{24}$

Angesichts dieser biologischen »Fluidität « bemüht sich Polemon darum, das Bild des Männlichen in strikter Opposition zum Weiblichen zu normieren. ${ }^{25}$ Das biologische Geschlecht ist dabei nebensächlich:

»Nun werde ich die Kennzeichen für männliches und weibliches Aussehen (signa formae maris et feminae) und ihre physiognomische Bedeutung darlegen. Du wirst erkennen welche von beiden [in jedem einzelnen Individuum] vorherrscht und das Ergebnis wird dich in deinem Urteil leiten (utrum prae altero valeat observabis et eo potissimam iudicii tui partem moderaberis). Das Weibliche hat, im Vergleich zum Männlichen, einen kleinen Kopf, einen kleinen Mund, weicheres dunkelfarbiges Haar, ein engeres Gesicht, hell glänzende Augen, einen engen Nacken, einen leicht schrägen Brustkasten, schwache Rippen, größere fleischigere Hüften, engere Schenkel und Waden, leichte X-Beine, zierliche Fingerspitzen und Zehen, der Rest des Körpers feucht und schlaff, mit weichen Gliedmaßen, die langsam entlang gleiten. Das Männliche jedoch ist in jederlei Hinsicht das Gegenteil zu dieser Beschreibung (sed mas huic descriptioni ex omni parte contrarius). Es ist jedoch möglich männliche Eigenschaften auch in Frauen zu finden (licet marium similitudinem in feminis quoque invenias)." (Physiogn. 2 [25r/25v]; Übers. nach Gleason $)^{26}$

Die Übereinstimmung zwischen diesen Beschreibungen und den öffentlichen Statuen von Göttern und herausragenden Gestalten ist augenfällig. Die Statuen dienten als visuelle Verstärker des Männlichkeitsdiskurses und stellten zugleich eine Beziehung zur Welt des Numinösen her.

\section{Mannsein, Kontrolle und Herrschaft ${ }^{27}$}

Der männliche Körper ist vollkommen, er ist nach außen gerichtet, warm, aktiv, stark, kontrolliert - damit ist er nach antikem Verständnis zur Herrschaft bestimmt.

23. Vgl. allgemein Gleason, a. a. O. (s. Anm. 2).

24. Gleason, a.a. O., 58 (ed. Förster, 192/8-14). Vgl. auch Physiogn. 2 [25r/25v] (Gleason, a.a. O., 60). Aus der Tierwelt leitet (Ps.-)Aristoteles, Physiog. B 5,809b4-13 ähnliche Unterschiede ab.

25. Ein sprechendes Beispiel bietet Seneca, Ep. mor. 114,3 (übers. Rosenbach): »Wenn sie [= die Seele] verweichlicht ist, wird allein schon im Gang die Weichlichkeit deutlich (si ille effeminatus est, in ipso incessu apparere mollitiam)."

26. Gleason, a. a. O., 60 (ed. Förster, 192/19-194/16).

27. »Herrschaft « fasse ich auf als die reale Möglichkeit zur dauerhaften Durchsetzung des eigenen 
Wenn es ein hervorstechendes Merkmal für Männlichkeit in der Antike gibt, dann ist es dieser Anspruch:

Klassisch formuliert Aristoteles: »Desgleichen ist das Verhältnis des Männlichen zum Weibli-

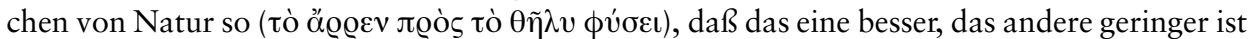

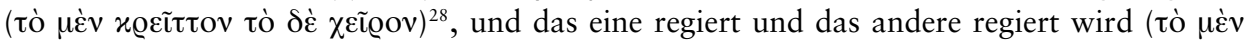

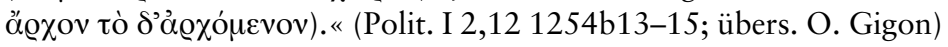

Dieses Ideal »hegemonialer Männlichkeit « bildet das Ende einer Skala, in der Herrschaft und Männlichkeit streng aufeinander bezogen sind..$^{29}$ Da der Zugang zu Herrschaft jedoch limitiert ist, stehen die »Anwärter « darauf in Konkurrenz zueinander. ${ }^{30}$ Innerhalb der sozialen Pyramide waren es daher insbesondere die Eliten, die Zugang zu Männlichkeit hatten. Ausgeschlossen waren alle, die per se als unmännlich galten: Frauen (nach anatomischem Maßstab), Kinder (auch Buben), Sklaven (jedweden Geschlechts), sexuell passive Männer, weibliche Männer (sog. effeminati), Eunuchen, "Barbaren ", usw. ${ }^{31}$ Innerhalb eines Lebenszyklus konnte das Maß an Männlichkeit variieren, so dass sich Männer in vorangeschrittenem Alter gegen die Gefahr der Unmännlichkeit wehren mussten:

»Von allen Übeln entehren keine einen alten Mann mehr als Müßiggang ( $\alpha \dot{\tau} \varrho \alpha \xi i ́ \alpha)$, Feigheit

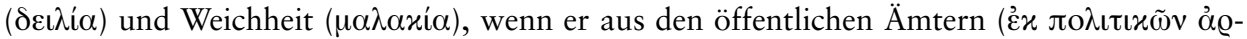

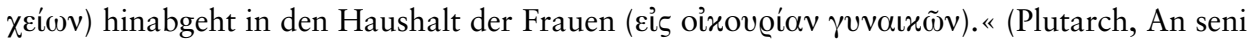
respublica gerenda sit, 784A; eig. Übers.)

$\mathrm{Ob}$ in der Politik, im Sport, im Krieg oder in der öffentlichen Rede, überall steigt mit zunehmender Aktivität und Kontrolle zugleich auch die Männlichkeit. ${ }^{32}$ Besonders deutlich ist dies auf dem Gebiet der Sexualität. Grundsätzlich wurde männliche Sexualität als Befriedigung eines natürlichen Verlangens wie Hunger oder Schlaf verstanden. ${ }^{33}$ Dieses Verlangen war erst dann ein moralisches Problem, wenn es der Kontrolle männlicher Rationalität zu entgleiten drohte. ${ }^{34}$ Die eigentlichen Sexualpraktiken waren vornehmlich Ausdruck bestehender Herrschaftsbeziehungen, wobei das spezifisch Männliche in der persönlichen Freiheit und Souveränität zum Ausdruck kommt. ${ }^{35}$ Die Ausübung von Herrschaft und die Wahrung der sozialen

Willens gegenüber einer anderen Person oder Personengruppe. "Kontrolle « meint die ständige Überprüfung bzw. Nachprüfung zur Sicherung der Macht. Da Herrschaft sehr unterschiedlich begründet sein kann (rational, legal, physisch, usw.), ist Kontrolle für das System sehr wichtig.

28. Ähnlich Josephus, C. Ap. 2,24: »Die Frau ist in jeder Hinsicht geringer als der Mann."

29. J. C. Anderson/S. D. Moore, Matthew and Masculinity, in: Moore/Anderson, a. a. O. (s. Anm. 7), $68 \mathrm{f}$.

30. T. B. Liew, Re-mark-able Masculinities. Jesus, the Son of Man, and the (sad) Sum of Manhood?, in: Moore/Anderson, a.a.O., $104 \mathrm{f}$.

31. Anderson/Moore, a. a. O. (s. Anm. 29), 69.

32. T. Späth, Männerfreundschaften - politische Freundschaften? Männerbeziehungen in der römischen Aristokratie des Prinzipats, in: W. Erhardt/B. Herrmann (Hg.), Wann ist der Mann ein Mann?, Stuttgart 1997, 195. Alston, a.a. O. (s. Anm. 5), 207-209 sieht einen engen Zusammenhang zwischen libertas und potestas.

33. Vgl. Xenophon, Symp. 4,38; Memorabilia I 3,14f.; II 1,30; IV 5,9; IV 5,9; zum Kyniker Diogenes s. Diog. L., VI 2,46.69; Dion von Prusa, Reden 6,17-20; Galenos, De locis affectis 6,5. Vgl. M. Foucault, Der Gebrauch der Lüste. Sexualität und Wahrheit 2, Frankfurt a. M. 1986, 71-83.

34. Foucault, a.a. O., 64: »Der Exzeß und die Passivität sind für einen Mann die beiden Hauptformen der Immoralität in der Praktik der aphrodísia."

35. Vgl. das Ergebnis der erhellenden Studie von Meyer-Zwiffelhoffer, a.a. O. (s. Anm. 21), 213. 
Unterschiede waren daher wesentlich bedeutsamer als der heute so ausgiebig diskutierte Gegensatz zwischen Homo- und Heterosexualität. ${ }^{36}$

Die Tatsache, dass Kontrolle und Herrschaft als wichtigste Ausdrucksformen des Männlichen galten, erlaubte eine philosophische Verlagerung von Männlichkeit in das Innere des Menschen. ${ }^{37}$ Besonders Männer, denen der Zugang zur äußeren Herrschaft der römischen Eliten verwehrt war, konnten ihr Mannsein in Form von Selbstkontrolle, Mäßigung und Willensstärke unter Beweis stellen. ${ }^{38}$ Die hellenistischen Moralisten haben Gier, Lust und fehlende Selbstbeherrschung mit dem Weiblichen in Verbindung gebracht. Entbehrung, Strenge und Selbstkontrolle galten hingegen als Zeichen von Männlichkeit. ${ }^{39}$ Die höchste Form von Männlichkeit käme demnach dort zum Ausdruck, wo sich jemand im Hinblick auf Ärger, Lust und v. a. auf sexuelles Begehren selbst unter Kontrolle hat. In der römischen Stoa finden wir diese Auffassung etwa bei Seneca, Musonius Rufus und seinem Schüler Epiktet. ${ }^{40}$

\section{Aspekte von Männlichkeit in der Korintherkorrespondenz ${ }^{41}$}

\section{Angefochtene Männlichkeit (2 Kor)}

Der 2. Korintherbrief bietet einen geeigneten Einstieg in die Thematik, weil der Konflikthintergrund zwischen Paulus und seinen Gegnern u. a. auch den antiken Männlichkeitsdiskurs berührt. ${ }^{42}$ Die Vorwürfe, die gegen Paulus erhoben werden, betreffen sein öffentliches Auftreten und Aspekte seines persönlichen Charakters:

36. Vgl. C. A. Williams, Roman Homosexuality. Ideologies of Masculinity in Classical Antiquity, New York/Oxford 1999. Aristoteles, Probl IV 26,879a36-880a5 behandelt ausführlich die Frage: "Warum haben einige am Geschlechtsverkehr Freude, und zwar die einen (von ihnen), indem sie dabei den aktiven Teil spielen, die anderen aber, (indem sie dies) nicht (tun, sondern passiv sind)? « (übers. Flashar)

37. Vgl. in diesem Sinne die Titel von Foucaults unabgeschlossener Geschichte der Sexualität: Für Griechenland »Der Gebrauch der Lüste« (Bd. 2) und für Rom »Die Sorge um sich « (Bd. 3).

38. Diese Option war z. B. für die politisch geschlagenen Juden wichtig; vgl. zu 4Makk die Studie von S. D. Moore/J. C. Anderson, Taking it Like a Man. Masculinity in 4 Maccabees, in: JBL 117 (1998), 249-273; und zum rabbinischen Judentum M. L. Satlow, »Try to be a Man «. The Rabbinic Construction of Masculinity, in: HThR 89 (1996), 19-40. Wenn z. B. Sokrates gegenüber dem aufbrausenden Sophisten Polos darlegt, dass Unrechtleiden besser ist als Unrechttun und dass es gut und schön ist, für ein Unrecht bestraft zu werden (Platon, Gorgias 468e-479e), dann stehen sich hier zwei Männlichkeitskonzepte gegenüber: die herrschende Version, die auf Mut, Gewalt und Herrschaft gründet, und die philosophische Version, die auf Selbstdisziplin, Durchhaltevermögen und Selbsthingabe setzt. Sokrates stellt damit die moralische Vernunft über die männliche Herrschaftsvorstellung (v. a. gegenüber Kallikles): »Denn das Sterben selbst fürchtet ja wohl nie-

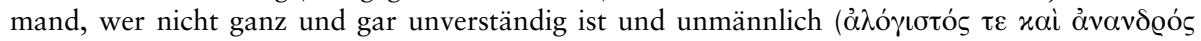
$\grave{\varepsilon} \sigma \tau \iota v)$; das Unrechttun aber fürchtet man. Denn mit vielen Vergehungen die Seele angefüllt, in die Unterwelt kommen, ist unter allen Übeln das ärgste. « (522e, übers. Schleiermacher)

39. Vgl. E. Dench, Austerity, Excess, Success, and Failure in Hellenistic and Early Imperial Italy, in: Wyke, a.a.O. (s. Anm. 2), 121-146.

40. Den Unterschied zu den Peripatetikern betont Seneca selbst in Ep. mor. 116,1.

41. Vgl. allgemein zum Thema bei Paulus D. J. A. Clines, Paul, the Invisible Man, in: Moore/Anderson, a.a. O. (s. Anm. 7), 181-192; J. Larson, Paul's Masculinity, in: JBL 123 (2004), 85-97; Leutzsch, a.a.O. (s. Anm. 8).

42. Vgl. Larson, a.a. O.; J. A. Harrill, Invective against Paul (2 Cor 10:10), the Physiognomics of the Ancient Slave Body, and the Greco-Roman Rhetoric of Manhood, in: A. Yarbro Collins/M. M. 
Als öffentlicher Redner konnte sich Paulus dem Forum männlicher Selbstdarstellung und Kompetitivität kaum entziehen. Gemessen an professionellen Standards konnten bereits unscheinbare Gesten und physiognomische Eigenheiten zu Missverständnissen führen. ${ }^{43}$ Der Vorwurf unmännlichen Auftretens war ein beliebter polemischer Topos ${ }^{44}$ und die Gegner des Paulus haben darauf zurückgegriffen (2 Kor

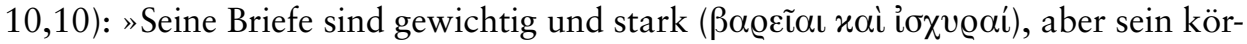

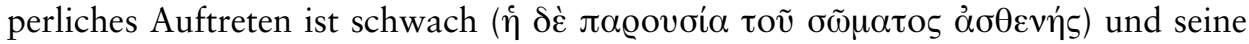

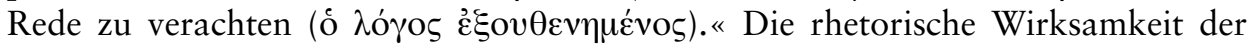
Briefe steht - selbst für seine Gegner - ganz außer Frage, die »performance « lässt jedoch zu wünschen übrig. ${ }^{45}$ Der öffentliche Körper des Paulus ist nach den geltenden Standards "schwach ${ }^{46}$ und damit ist auch seine Rede verachtens- und vernachlässigenswert. ${ }^{47}$ Vor dem Hintergrund obsessiver männlicher Körperkontrolle wird deutlich, dass es den Gegnern darum ging, die mannhafte physische Erscheinung des Paulus herabzuwürdigen und damit zugleich auch seinen Charakter, seine apostolische Autorität und die Glaubwürdigkeit des von ihm verkündigten Evangeliums in Frage zu stellen. Die Botschaft von Gottes Heil kann aus dieser Perspektive nicht von einem physisch » minderwertigen « Mann verkündigt werden. ${ }^{48}$

Mit dem Körper gerät auch der Charakter des Paulus ins Zentrum der Kritik. Offensichtlich haben die Gegner Änderungen seiner Reisepläne zum Anlass genommen, um ihm Leichtsinn, Wankelmut, Unzuverlässigkeit und Rückgratlosigkeit (1,17a: $\left.\varepsilon^{2} \lambda \alpha \phi \varrho i ́ \alpha\right)$ vorzuwerfen. ${ }^{49}$ Paulus verteidigt sich (1,17f.): »Indem ich mir dies nun vornahm, habe ich etwa Leichtfertigkeit ( $\dot{\varepsilon} \lambda \alpha \phi \varrho i \alpha)$ walten lassen? Oder nehme

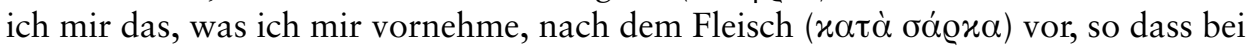
mir das Ja-ja zugleich das Nein-nein wäre ${ }^{50}$ Gott aber ist treu, dass unser Wort an

Mitchell (Hg.), Antiquity and Humanity. Essays on Ancient Religion and Philosophy (FS H. D. Betz), Tübingen 2001, 189-213.

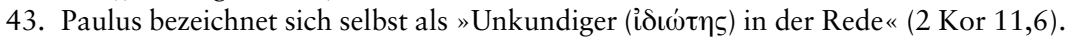

44. Vgl. z. B. Dio Cass. 46,18,4-6; Tacitus, Dial. 18,5.

45. M. E. Thrall, Commentary on II Corinthians, Vol. 2: VIII-XIII (ICC), Edinburgh 2000, 632 sieht jedoch einen Widerspruch, wenn es um den rhetorischen Vollzug geht: »In that case, however, why should there be so strong a contrast with the letters? « Weil es in der antiken Rhetorik (auch) um »Selbstrepräsentation « geht, ist der Unterschied zwischen geschriebenem Text und »Aufführung « sehr hoch zu bewerten.

46. Ein Bezug zum »niederen « Handwerk des Paulus ist m. E. hier nicht im Blick (vgl. zur Diskussion Thrall, a. a.O., 631f.).

47. Vgl. zur physisch starken Konstitution des Redners P. Marshall, Enmity in Corinth. Social Conventions in Paul's Relations with the Corinthians (WUNT 2:23), Tübingen 1987, 384-390. Seneca Controversiae 3, pref 2 lobt die Rhetorik des Cassius Severus als stark und beeindruckend (oratio eius erat valens, culta, vigentibus plena sententiis). Das Verb $\mathfrak{c}_{\xi} \xi 0 v \theta \varepsilon v \varepsilon \dot{\varepsilon} \omega$ bedeutet »verachten « (z. B. eine andere Person: Lk 18,9; Röm 14,3.11 [zusammen mit »richten «]; 1 Kor 16,11; Gal 4,14), »demütigen « (Lk 23,11: Verspottung Jesu); "als gering achten ", "nicht als gültig anerkennen « (Apg 4,11; 1 Kor 6,4; 1 Thess 5,20). In diesem Sinne hat Gott die »Verachteten «, die Armen und Unedlen, auserwählt (1 Kor 1,28).

48. Man darf diese Haltung wohl als pneumatologischen »Machismo« bezeichnen.

49. Griech. ع̇̀ $\alpha \phi \varrho i ́ \alpha$ ist nicht häufig belegt. Sachlich lässt sich über die lateinische Entsprechung levitas der Hintergrund dieses Vorwurfs beleuchten (vgl. Cicero, Phil. 7,9; Acad. 2,66.120; Brutus 27,103; Off. I,26,90; Fin. 1,61; Tusc. 1,40,95; Caesar, Bell. 2,1; Quint. 10,3,17).

50. Der Sinn von tò Naì vai xai tò Ov̉ oủ $(1,17 \mathrm{~b})$ ist umstritten (vgl. Thrall, a.a. O. [s. Anm. 45], 140-143). M.E. ergibt sich von 1,18 (Nai xai Ov̉) her ein kohärentes Verständnis für 1,17b im Sinne eines gleichzeitigen Ja und Nein (vgl. E. Gräßer, Der zweite Brief an die Korinther, Bd. 1 [ÖTBK 8], Gütersloh 2002, 74-76). Der Sinn wird im Zusammenhang mit dem Vorwurf der Leichtfertigkeit klarer. 
euch nicht Ja und Nein zugleich ist. «Im Rückblick auf einen wenig erfreulichen Zwischenbesuch greift Paulus wieder die Sprache seiner Gegner auf und sagt von sich selbst: "Anwesend unter euch zwar unterwürfig ( $\tau \alpha \tau \varepsilon \iota v o ́ \varsigma)$, abwesend aber bin ich euch gegenüber mutig $(\theta \alpha \varrho \varrho \tilde{\omega})$. $(10,1 \mathrm{~b})$ Darin spiegelt sich der Vorwurf wider, Paulus sei in der direkten Konfrontation feige. Vielleicht haben auch eigene frühere Aussagen zu solch negativen Einschätzungen Anlass gegeben: Paulus sagt von sich, dass er sich »allen zum Sklaven « mache (1 Kor 9,9-23) und dass er versuche, » in allen Dingen allen zu gefallen « (1 Kor 10,33). Das sind Verhaltensweisen, die gerne dem charakterlosen Schmeichler zugesprochen wurden. ${ }^{51}$ Als unterwürfiger Opportunist, falscher Freund von Vielen und hündischer Feigling stand die Figur des Schmeichlers in einem klaren Widerspruch zum römischen Männlichkeitsideal. ${ }^{52}$ Für die korinthischen Gegner gab es wohl noch andere Anzeichen für die Limitierung männlicher Autonomie seitens des Apostels: So verdiente er z. B. seinen Lebensunterhalt von seiner eigenen Arbeit als Handwerker. Auch musste er mehrmals die Strafe der Auspeitschung erleiden (2 Kor 11,23-25). Es stellt sich zudem die Frage, wie sie das Weinen des Apostels (Stichwort: Tränenbrief) eingeschätzt haben $(2,4)$. Die römische Stoa hatte für derlei Gefühlsausdrücke nicht viel übrig.

Angesicht solcher Vorwürfe ist die Verteidigungsstrategie des Apostels in ihrer Zuspitzung auf Aspekte von Männlichkeit zu betrachten. Bereits in 1 Kor 4,21 nennt

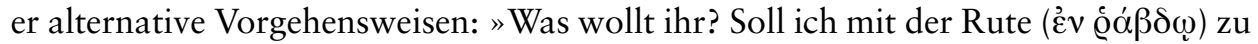

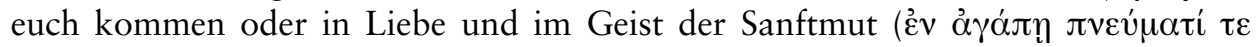

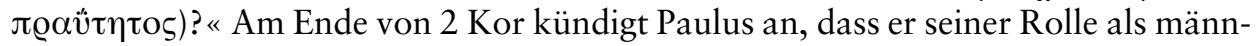
liche Leitfigur durchaus nachkommen könnte $(13,2)$ : »Ich habe es vorausgesagt und sage es abermals voraus wie bei meinem zweiten Besuch, so auch nun aus der Ferne denen, die zuvor gesündigt haben, und allen übrigen: Wenn ich noch einmal komme,

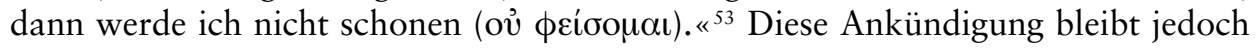
merkwürdig unbestimmt. Trotz der angespannten Situation hat die Reaktion des Paulus in 2 Kor nach den geltenden Männlichkeitsstandards nicht viel zu bieten. Er appelliert vielmehr bei der Eröffnung seiner Verteidigungsrede an die »Sanftmut und

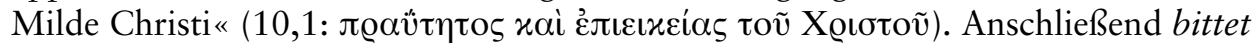
er darum, dass er " anwesend nicht mutig sein muss « $(10,2)$. Unterlegt mit leichter Ironie, erinnert er die Korinther daran, dass er und seine Mitarbeiter - im Gegensatz zu seinen Gegnern - einfach zu »schwach « waren, um die Gemeinde zu »unterdrücken « (11,20f.). Ob sich Paulus über einen Mangel an männlicher Repräsentanz bewusst war, lässt sich kaum sagen. Es ist jedoch auffällig, dass er das Verhältnis gegenüber den Korinthern in Metaphern fasst, die aus der Werkstatt römischer Männlichkeit stammen: Er ist Vater der Gemeinde (1 Kor 4,14f.; 2 Kor 11,2 f.; 12,14), er ist geistlicher Soldat (2 Kor 10,3-5) und siegreicher Athlet (1 Kor 9,2427). ${ }^{54}$

51. Vgl. zum polemischen Topos der Schmeichelei Marshall, a.a. O. (s. Anm. 47), 281-325. Der technische Begriff $\varkappa 0 \lambda \alpha x \varepsilon i \alpha$ erscheint nur in 1 Thess 2,5.

52. Vgl. allgemein Marshall, a.a. O., 70-90 (zum Vorwurf der Unmännlichkeit: 78, Anm. 48) und Larson, a.a. O. (s. Anm. 41), 92.

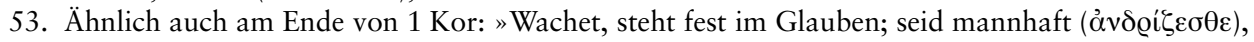
seid stark!« $(16,13)$

54. Auf der anderen Seite lässt sich sein Umgang mit den Korinthern kaum als » autoritär « bezeichnen und Paulus gebraucht ebenso auch Bilder aus dem Feld der Mütterlichkeit; vgl. B. R. Gaventa, Our Mother St. Paul. Toward the Recovery of a Neglected Theme, in: A.-J. Levine (Hg.), A Feminist Companion to Paul, London 2004, 85-97. 
Worauf gründet Paulus seine Verweigerung, sich auf das »Spiel« der Gegner einzulassen? Der Hinweis auf die "Sanftmut und Milde Christi « $(10,1)$ ist keine rhetorische Beiläufigkeit, sondern bezeichnet (mit $\delta \iota \alpha)$ den Ermöglichungsgrund für die moralische Ermunterung bzw. Ermahnung des Apostels. ${ }^{55}$ Die angeführten »Tugenden « Jesu sind leitend für den Umgang mit den Korinthern und erscheinen dadurch auch als Alternative zum römischen Männlichkeitsideal. Eine tiefergehende theologische Grundlegung für dieses Verhalten lässt sich dem kreuzestheologischen Entwurf in Kor 1-2 entnehmen. Hier bindet Paulus den Erweis göttlicher Macht an die Schwäche des Kreuzes und lässt korinthische Wertvorstellungen von Weisheit und Rhetorik am Kreuzesparadoxon zerbrechen. Dies prägt das Verhältnis des Paulus zu den Korinthern: Wenn Stärke und Schwäche auf so komplexe Art und Weise ineinandergreifen, dann kann der Apostel nicht aus einer Position männlicher Herrschaft heraus operieren. Es liegt in der Konsequenz eines solchen Verständnisses, dass er sich aus dem kompetitiven Feld öffentlicher Rede zurückzieht.

\title{
2. Männlichkeit unter Konstruktion (1 Kor)
}

Der antike Männlichkeitsdiskurs ragt nicht nur in das komplexe Beziehungsgeflecht zwischen Paulus und den Korinthern hinein, er spiegelt sich auch in der paulinischen Paränese wider. Exemplarisch sollen daher einige Texte aus 1 Kor 6-11 betrachtet werden:

a) Paulus gegen »unmännliche Männer«

In 1 Kor 6,9 spricht Paulus eine deutliche Warnung gegen die Ungerechten aus, die das » Reich Gottes nicht erben werden «. Neben Unzüchtigen, Götzendienern und Ehebrechern werden auch $\mu \alpha \lambda \alpha x$ oí und $\alpha \varrho \sigma \varepsilon v o x$ ĩ̃ $\alpha$ l dazu gerechnet. Die meisten deutsch-

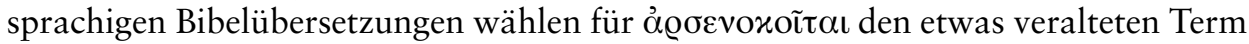
»Knabenschänder «. Die genaue semantische Bestimmung, die hier nicht weiter verfolgt werden kann, ist jedoch alles andere als eindeutig, zumal das Wort äußerst selten belegt ist. ${ }^{56}$ Das Übersetzungsangebot für $\mu \alpha \lambda \alpha x o i$, das bis zum Beginn des 20. Jhs. mit » Weichlinge « übersetzt wurde, hat sich in letzter Zeit erweitert: ${ }^{57}$

\author{
Lustknabe Luther rev. (1975, 1984), Einheitsübersetzung (1979) \\ Lüstling U. Wilckens (Benziger, 1970), J. Kürzinger (Pattloch, 1953) \\ Strichjunge K. Berger (Insel, 1999, 92) \\ Weichlinge Luther (1534), Luther rev. NT (1956), Schlachter rev. (1981, 2000) \\ Wollüstlinge Elberfelder NT (1975)
}

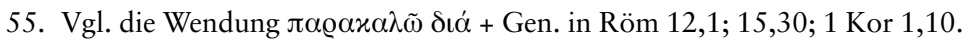

56. Vgl. zur Diskussion D. B. Martin, Arsenokoitês and Malakos: Meanings and Consequences, in: R. L. Brawley (Hg.), Biblical Ethics and Homosexuality. Listening to Scripture, Louisville 1996, 117-136:118-123; W. L. Petersen, Can Arsenokoitai be translated by >Homosexuals<?, in: VigChr 40 (1986), 187-191; D. F. Wright, Homosexuals or Prostitutes? The Meaning of Arsenokoitai (1 Cor. 6:9, 1 Tim. 1:10), in: VigChr 38 (1984), 125-153.

57. Die stärker paraphrasierenden Übersetzungen gehen hier z. T. recht kreative Wege: »mit Partnern aus dem eigenen Geschlecht verkehren « (Gute Nachricht, 1978); jemand, »der sich von seinen Begierden treiben lässt und homosexuell verkehrt « (Hoffnung für Alle, 1996); »Menschen, die als Männer mit Knaben oder ihresgleichen verkehren« (Gute Nachricht rev., 1997). 
Diese Vorschläge spiegeln zum Teil wechselnde Trends in der Wahrnehmung sexueller Fehlhandlungen wider. ${ }^{58}$ In seiner konventionellen Bedeutung bezieht sich das Adjektiv $\mu \alpha \lambda \alpha x o ́ s$ auf etwas Weiches wie Kleider, feine Speisen oder einen Windhauch. ${ }^{59}$ Im moralischen Diskurs behält das Wort einen Bezug zu solchen Negativeigenschaften bei, die als »weich « wahrgenommen werden: Faulheit, Verkommenheit, Feigheit, Genusssucht, Dekadenz oder, um all diese Laster in einer Kategorie zusammenzufassen, das » Weibliche $"{ }^{60}$ In sexuellen Kontexten kann $\mu \alpha \lambda \alpha x o ́ \varsigma$ sich auf einen Mann beziehen, der eine sexuell passive Rolle einnimmt. ${ }^{61}$ Ferner war dieser Zustand auch eine Frage der Physiognomie. Denn hier entschied sich, ob jemand mehr oder weniger »Mann « ist. ${ }^{62}$ Insgesamt scheint die alte Übersetzung » Weichlinge « sprachlich exakter zu sein als viele Optionen jüngeren Datums.

Eine ähnlich negative Einschätzung findet sich in der verworrenen Argumentation über die Geschlechterordnung im Gottesdienst in 1 Kor 11,2-16. Der ideale Mann ist in diesem Text weiterhin der Frau vorgeordnet und in seiner herrschenden Funktion näher an der göttlichen Herrlichkeit als die Frau (11,2-10). ${ }^{63}$ Paulus zielt auf eine öffentliche Darstellung von Männlichkeit, die jede Überschneidung in der symbolischen Repräsentation der Geschlechter meidet. Während er Männern gebietet, nicht mit langem Haar in der Versammlung zu sprechen $(11,14)$, sollen die Frauen nicht mit offenem Haar auftreten (11,4-7). ${ }^{64}$ Die Abneigung gegen langhaarige Männer bewegt sich auf der gleichen Ebene wie der Ausschluss der weiblichen Männer aus dem Himmelreich. ${ }^{65}$ Das einzige Argument das Paulus jedoch hierfür anzuführen vermag, ist der Rekurs auf die konventionellen Werte von Ehre und

58. Ein Blick in die englischen Bibelübersetzungen zeigt ein ähnliches Bild: »effeminate (KJV 1611, ASV 1901), "male prostitutes « (NRSV 1989, NIV), »homosexual perversion « (RSV 1946, TEV 1966, NEB 1970, REB 1992). W. Bauer/K. Aland/B. Aland, Griechisch-deutsches Wörterbuch zu den Schriften des Neuen Testaments und der frühchristlichen Literatur, Berlin/New York ${ }^{6} 1988$, 991: »Lustknaben, Männer und Jünglinge, die sich mißbrauchen ließen «. Eindeutiger drückt sich J. P. Louw/E. A. Nida, Greek-English Lexicon of the New Testament based on semantic domains, New York 1989, 88.281 aus: » The passive male partner in homosexual intercourse - 'homosexual..«

59. Vgl. Martin, a.a. O. (s. Anm. 56), 124-128. Mt 11,8 // Lk 7,25 (»weiche Kleider «) impliziert vielleicht Vorurteile gegen »unmännliche« Männer in den höheren Regierungsschichten.

60. Vgl. Josephus, BJ 7,338; Ant 5,246; 10,194; weitere Belege in Martin, a. a. O., 124-127.

61. Es kann auch jemanden bezeichnen, der auf Männer wie Frauen begehrenswert wirkt. Vgl. zum effeminatus den materialreichen Artikel von H. Herter, Art. Effeminatus, in: RAC 4 (1959), 620650.

62. Vgl. (Ps-)Aristoteles, Physiogn 808a10.34 (übers. S. Vogt).

63. Die Frage, ob $x \varepsilon \phi \alpha \lambda \eta \dot{~ i n ~ 11,3 ~ i m ~ S i n n e ~ v o n ~ » U r s p r u n g ~ « ~ o d e r ~ i m ~ S i n n e ~ v o n ~ » H a u p t ~ « ~ z u ~ v e r s t e h e n ~}$ ist, wird kontrovers diskutiert, ändert aber kaum etwas an der Rolle des Mannes als Agens gegenüber der passiven Frau.

64. Vgl. zur hier zugrunde liegenden Deutung D. W. J. Gill, The Importance of Roman Portraiture for Head-Coverings in 1 Corinthians 11:2-16, in: TynB 41 (1990), 245-260; J. Murphy-O’Connor, Sex and Logic in 1 Cor 11:2-16, in: CBQ 42 (1980), 482-500; R. E. Oster, When Men Wore Veils to Worship. The Historical Context of Cor 11.4, in: NTS 34 (1988), 481-505; C. L. Thompson, Hairstyles, Head-coverings, and St. Paul. Portraits from Roman Corinth, in: BA 51 (1988), 99115.

65. Vgl. Ps-Phokylides, 210-212; weitere Belege in Murphy-O’Connor, a. a. O., 485-487. Chrysostomus deutete bereits diesen Text als Hinweis auf langes Haar (In Epistulam 1 ad Corintios hom. 26,1 = PG 61, 213). 
Schande. ${ }^{66}$ Damit steht er wieder fest auf dem Boden hegemonialer römischer Männlichkeit. ${ }^{67}$

Es ist gut vorstellbar, dass die Eliten innerhalb der korinthischen Gemeinde zu einem Lebensstil neigten (vgl. 1 Kor 11,17ff.), der als »Verweiblichung « gewertet werden konnte - zumal es Hinweise gibt, dass die korinthischen Männer generell einen solchen Ruf genossen. Das sei mit einem kurzen Epigramm Martials veranschaulicht:

»Da du dich als Landsmann der Korinther rühmst (municipem Corinthiorum iactes), Charmenion, was keiner bestreitet, weshalb nennst du mich dann >Bruder $<$, wo ich doch von Keltiberern stamme und Bürger des Tagus bin? Sehen wir uns etwa im Gesicht ähnlich? Du läufst pomadisiert mit onduliertem Haar herum, ich widerspenstig mit meinem spanischen Schopf; du glatt von der täglichen Enthaarungsprozedur, ich mit stachligen Beinen und Wangen; dein Mund lispelt, und schwach ist deine Zunge, bei mir reden kräftiger selbst die Gedärme noch: 'So unähnlich ist nicht die Taube dem Adler oder die flüchtende Gazelle dem grausamen Löwen. 'Hör' daher auf, mich >Bruder` zu nennen, sonst nenn' ich dich, Charmenion, `Schwester $\iota$ ! (Martial, Epigr. X 65; übers. Barié / Schindler, 733) ${ }^{68}$

Für die paulinische Vorstellung von Männlichkeit wäre ein solcher »Charmenion « eine Schande für den christlichen Gottesdienst. ${ }^{69}$

b) Beschränkungen männlicher Stärke und Autonomie

Die Diskussion in 1 Kor 8-10 um das Problem des Götzenopferfleisches nimmt Paulus zum Anlass, um Freiheit und Liebe zueinander in Beziehung zu setzen. ${ }^{70}$ Die Haltung derer, die sich von den »Schwachen « absetzen, lässt sich problemlos in das Netz hegemonialer Männlichkeit einschreiben. Durch die autonome Entscheidung, ohne jede Rücksicht Opferfleisch zu konsumieren, erweisen sie sich als wahrhaft mannhaft. Paulus stellt ihre moralische Integrität nicht in Frage. Seine Vermittlungsversuche zielen jedoch darauf ab, dass sie ihre Gewissens- und Handlungsfreiheit den konkreten Nöten der schwächeren Brüder unterordnen (8,9): »Seht aber zu,

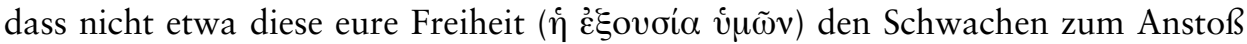

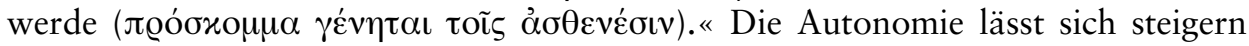
zur Freiheit auf Freiheitsverzicht aus Liebe zu einem höheren gemeinschaftlichen

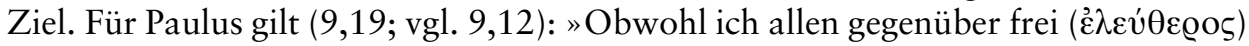

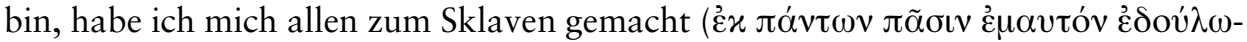
$\sigma \alpha)$, damit ich möglichst viele gewinne. « Der Athlet, Paradigma für Männlichkeit,

66. Das semantische Feld von Ehre und Schande liegt der gesamten Passage zugrunde: sein Haupt

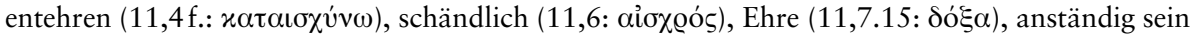

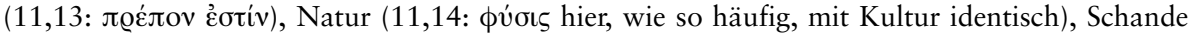

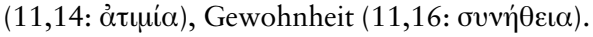

67. Leutzsch, a.a. O. (s. Anm. 8), 607 nennt diese Position »Differenzandrozentrismus «.

68. Ähnlich Juvenal, Satir. 8,113-120: »Vielleicht magst du die unkriegerischen Rhodier und das parfümierte Korinth mit Recht verachten: was könnten dir schon die enthaarte Jugend und die glatten Beine des ganzen Stammes anhaben? Das rauhe Spanien muß man meiden, das gallische Land und die Küsten Illyriens ... (übers. Adamietz, 175)

69. Es wäre reizvoll, diesen kategorischen Ausschluss mit dem Wort Jesu über die »Kastraten, die sich für das Himmelreich selbst kastriert haben « (Mt 19,12) zu vergleichen.

70. Vgl. zum Verhältnis von $\mathfrak{\varepsilon} \xi o v \sigma i ́ \alpha$ und $\dot{\varepsilon} \lambda \varepsilon v \theta \varepsilon \varrho i \alpha ~(8,9 ; 9,1.4$ f.12.18f.) Marshall, a.a.O. (s. Anm. 47), 285-289. Beide stehen in enger Beziehung zum lat. libertas. Zur Intervention des Paulus in 1 Kor 8-10 vgl. M. Mayordomo, Paul as Mediator, in: F. Enns u. a. (Hg.), Seeking Cultures of Peace. A Peace Church Conversation, Telford 2004, 171-181. 
dient Paulus als Beispiel für zweckgerichteten Freiheitsverzicht (9,24-27). Zwei Männlichkeitskonzepte stehen sich damit gegenüber: selbstbestimmte Stärke und Selbstverleugnung aus Liebe.

c) Männliche Identität, Sexualität und Zugehörigkeit zu Christus

In 1 Kor 6,12-7,40 kommen Aspekte männlicher Identität und Sexualität zur Sprache. Paulus und die korinthischen Männer gehen jedoch von unterschiedlichen Konstruktionen aus. Für die römisch geprägten Männer in Korinth ist zu vermuten, dass Sexualität im Sinne von Befriedigung und Kontrolle (s.o.) verstanden wurde. Für Paulus sind drei Voraussetzungen maßgeblich: Erstens, auf dem Hintergrund alttestamentlich-jüdischer Anthropologie betrachtet er den Menschen als eine Einheit von körperlicher und nicht-körperlicher Identität. Zweitens, die biblische Schöpfungsgeschichte legt ein Verständnis von menschlicher Sexualität nahe, bei dem Mann und Frau durch einen Akt des Erkennens »ein Fleisch" werden (vgl. Gen 2,24 in 1 Kor 6,16). Schließlich, die Einwohnung des göttlichen Pneuma impliziert völlige Zugehörigkeit zum Herrn, nicht nur im Hinblick auf die innere SeelePerson, sondern auch im Hinblick auf die äußere Körper-Person. Den Korinthern hält Paulus entgegen, dass der Körper dem Herrn gehört (6,13b) und ein Glied Christi ist (6,15a), dass der Christ »einen Geist « mit dem Herrn bildet $(6,17)$ und schließlich dass der Körper » Tempel des Heiligen Geistes « ist (6,19a). Diese körperliche Dimension gründet in der Überzeugung, dass Gott Jesus körperlich von den Toten auferweckt hat (6,14; vgl. Kap. 15). Der männliche Körper ist damit keine moralisch neutrale Sphäre.

Zwischen Paulus und den Korinthern stehen weiterhin drei Fälle zur Debatte: Sexualverkehr mit einer Prostituierten, Sexualverkehr in der Ehe und Ehelosigkeit.

Der Fall der Prostituierten (1 Kor 6,12-20): Die Selbstverständlichkeit, mit der manche Männer der korinthischen Gemeinde zu Prostituierten gehen, lässt erkennen, dass für sie der Beischlaf mit einer untergeordneten, rechtlosen Frau eine legitime Form der körperlichen Befriedigung darstellte. Paulus nimmt Bezug auf ein Argument, das sehr gut zur korinthischen Position passt: »Die Speisen [sind] für den Bauch und der Bauch für die Speisen « (6,13a). Die korinthischen Männer stellten Speisen und Sexualität auf eine gleiche Ebene, weil sie nur den Körper und nicht das wahre Ich, die Seele, tangieren. Dem männlichen Motto »Alles ist mir erlaubt « $(6,12 a)$ setzt Paulus u. a. die Einschränkung entgegen: »ich werde mich durch nichts beherrschen lassen « $(6,12 \mathrm{~b})$. Wenn aus korinthischer Sicht diese Form von Sexualität ein Zeichen männlicher Freiheit und Herrschaft ist, dann zelebriert Paulus männliche Herrschaft im Modus der Selbstkontrolle. Die korinthische Praxis ist demnach von einem Widerspruch geprägt: Der sexuell aktive Mann ist zugleich passives Opfer seiner Leidenschaften. In der Ausübung männlicher Herrschaft verliert er demnach an Männlichkeit.

Ferner wird der männliche Körper, der durch das Pneuma zum auferstandenen Herrn gehört, im Sexualakt »ein Körper « mit der Prostituierten (6,16). Diese Form sexuellen Fehlverhaltens ist für Paulus nicht deswegen schlecht, weil sie einer anderen Person schadet ${ }^{71}$ - die menschliche Würde der Prostituierten hat Paulus (leider)

71. Musonius 12, Z. 22-28 entgegnet auf ähnliche Weise dem Argument, dass der Verkehr mit einer Prostituierten niemandem schade: » Gewiß sagt wohl einer, >aber so schwer wie der Ehebrecher sich gegen den Ehemann der verführten Frau versündigt, so schweres Unrecht tut doch niemandem 
nicht im Blick -, sondern weil sie sich am eigenen Leib als Ort der Beziehung zum auferstanden Christus verfehlt (6,18). Die Vorstellung, dass ein freier Mann und eine Prostituierte »ein Fleisch « und »ein Körper " sein könnten, war aus römischer Perspektive sicherlich absurd. Dadurch schafft Paulus jedoch eine perfekte Antithese zur korinthischen Praxis: Wenn der männliche Körper eins mit Christus ist, kann er nicht eins mit der Prostituierten sein. Anstelle von Befriedigung und Kontrolle umreißt Paulus damit den männlichen Körper als eine Sphäre von Intimität und exklusiver Zugehörigkeit zum Herrn. Dadurch aber wird ein Aspekt männlicher Sexualautonomie beschränkt, denn Gottes Geist zu haben, bedeutet: »ihr gehört nicht mehr euch selbst « $(6,19 \mathrm{~b})$. Männliche Herrschaftshoheit ist somit nicht nur vorübergehend, sondern permanent einer höheren Macht untergeordnet.

Ehe und Sexualität / Ehelosigkeit: An dieser Stelle wäre der Gedankengang weiterzuspinnen. Wenn das Ideal christlicher Männlichkeit die Unabhängigkeit von sexuellem Begehren ist und wenn der Körper voll und ganz zum auferstandenen Herrn gehört, dann könnte sogar die Ehe zum Problem werden. Denn auch dort wird im Sexualakt der Körper dem Herrn »entzogen «. Manche Männer in Korinth, die wohl kaum regelmäßig Prostituierte aufsuchten, scheinen in diese Richtung gedacht zu haben: »Es ist gut für einen Mann, eine Frau nicht zu berühren. « $(7,1)$ Bis zum Ende der Argumentation in 1 Kor 7 wird nicht völlig klar, ob Paulus diese Haltung ablehnt oder ihr doch zustimmt. ${ }^{72}$ Die Ehe als der privilegierte Ort für die Entfaltung von Sexualität hat für Paulus lediglich einen Existenzgrund: Nicht etwa das Aufziehen von Nachkommen (was allgemein in der Antike galt), sondern die Prävention sexu-

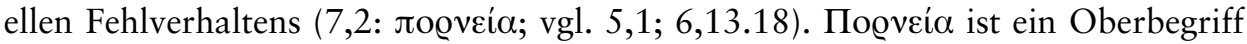
für den Bereich des sexuell Verbotenen und kann sich ganz allgemein auf »Unzucht « oder auch auf Prostitution beziehen. Es ist gut denkbar, dass Paulus und die Korinther sich darin einig waren, dass $\pi$ o@veí $\alpha$ zu meiden ist, dass sie aber nicht darin einig waren, was als solches zu werten ist. ${ }^{73}$ Paulus verortet das Problem im Inneren des Menschen, in der Versuchbarkeit aufgrund von Willensschwäche (in diesem Sin-

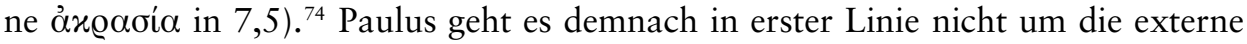
Realität von sexuellen Fehlhandlungen und Versuchungen, sondern um den inneren Mangel an Selbstkontrolle. In diesem Szenario ist die Ehe lediglich ein »Plan B «. Sie

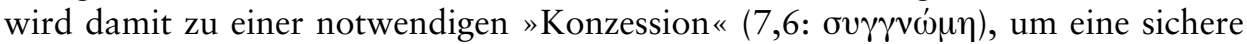

der Mann, der mit einer Dirne verkehrt oder, beim Zeus, mit einer Frau, die keinen Mann hat. Denn ein solcher Mann verdirbt doch niemandes Hoffnung auf (eheliche) Kinder.< Ich muß mit

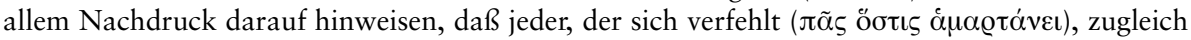

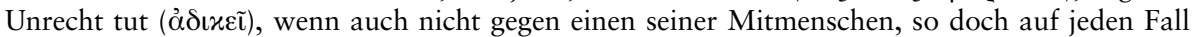
gegen sich selber, indem er sich schlechter und unsittlicher macht. « (übers. Nickel, $480 \mathrm{f}$.)

72. Vgl. zur Frage, ob 7,1b die Anfrage der Korinther enthält, H. Merklein, »Es ist gut für den Menschen, eine Frau nicht anzufassen «: Paulus und die Sexualität nach 1 Kor 7, in: G. Dautzenberg u. a., Die Frau im Urchristentum (QD 95), Freiburg i. Br. 1983, 225-253:229-232. In dieser absoluten Form stünde diese These in einem klaren Widerspruch zum zweiten Schöpfungsbericht (Gen 2,18: »Es ist nicht gut für den Menschen, dass er alleine sei! «), auf das 1 Kor 6 als Argument rekurriert.

73. Paulus muss z. B. betonen, dass mit der Duldung eines christlichen Mannes, der ein sexuelles Verhältnis mit der Stiefmutter unterhält, die Toleranzgrenze dessen, was selbst nach paganem Maß-

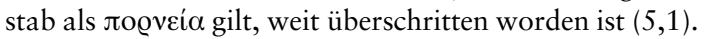

74. Die $\mathfrak{\alpha} x \varrho \alpha \sigma i \alpha$ ist ein zentraler Begriff der antiken Ethik. Es geht dabei um die Frage, warum Menschen, die das Gute kennen, dennoch gegen diese Erkenntnis handeln können (vgl. Platos Protagoras und die Behandlung des Problems in Aristoteles, NE 7,3). Damit wird das Problem der Willensschwäche berührt. 
Umgebung für männliches Kontroll-Management zu schaffen. »Es ist besser, zu heiraten, als [vor Verlangen] zu brennen.« $(7,9 \mathrm{~b})$

Innerhalb der Ehe anerkennt Paulus die Pflege der Sexualität als gegenseitige

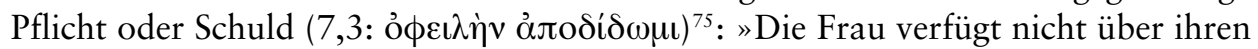
eigenen Leib, sondern der Mann; ebenso aber verfügt auch der Mann nicht über seinen eigenen Leib, sondern die Frau. « $(7,4)$ Nachdem Paulus in aller Deutlichkeit den männlichen Körper als »Tempel des Heiligen Geistes « in den Zugehörigkeitsbereich des auferstandenen Herrn gestellt hat, scheint dies eine bemerkenswerte Ausnahme zu sein. In der Ehe hat der Mann Kontrolle über den weiblichen Körper - eine Vorstellung, die ganz natürlich in das antike Männlichkeitskonzept passt aber, umgekehrt, verliert er das Verfügungsrecht über seinen eigenen Körper an die Frau. Der verheiratete Christ und die verheiratete Christin sind allerdings geteilt in ihrer Hingabe zu Gott (7,32-35). Das Problem ist dem Fall der Prostituierten nicht ganz unähnlich. Der große Unterschied liegt jedoch darin, dass die Ehe keine Sünde ist - ein Umstand den Paulus erstaunlicherweise dreimal hervorhebt (7,28a.28b.36).

Die Idealvorstellung des Paulus ist jedoch deutlich die, dass er Ehelosigkeit vorzieht (7,7-17.25-40), obwohl diese eine Gabe Gottes ist (7,7). Der Gegensatz zur Willensschwäche ist die Praxis der Enthaltsamkeit, die $\varepsilon^{3} \gamma \varkappa \varrho \alpha ́ \tau \varepsilon \iota \alpha(7,9)^{76}$, die in Gal 5,23 als "Gabe des Heiligen Geistes « aufgeführt wird (vgl. Tit 1,8; 2 Petr 1,6). Paulus gebraucht in 9,25 den Athleten als Vorbild für Enthaltsamkeit ( $\varepsilon \gamma \varkappa \varrho \alpha \tau \varepsilon v ́ o \mu \alpha \iota)$. Im Verlauf der Argumentation von 1 Kor 7 reicht er jedoch zwei vernünftige Argumente für die Ehelosigkeit nach: Erstens, angesichts der endzeitlichen Bedrängnisse werden Unverheiratete weniger Sorgen haben (7,26-28). Zweitens, Verheiratete sind geteilt in ihrer Hingabe (7,32-35). Mehrmals betont er, dass seine Absicht darin

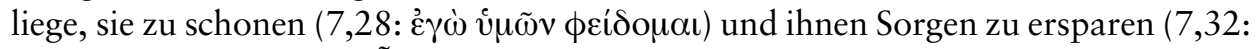

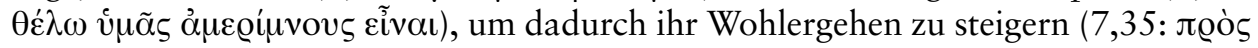

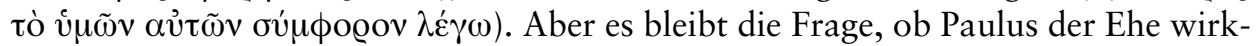
lich nur aus Sorge um die Endzeit skeptisch gegenüber steht. Das fundamentale Problem für Paulus scheint vielmehr im Mangel an Selbstkontrolle zu bestehen. Männlichkeit kulminiert in der kompletten Kontrolle über das sexuelle Verlangen. Diese anthropologische Vorgabe hat den Weg geebnet für die spätere Entwicklung asketischen Lebens von Männern wie Frauen. ${ }^{77}$ Aus dieser Perspektive waren die frühchristlichen Asketen keine defizitären Männer, sondern hypermaskuline Helden der Selbstkontrolle.

\section{Ergebnis}

Die Korintherbriefe bilden einen produktiven Ausgangspunkt, um einen paradigmatischen Einblick in die Konstruktion von Männlichkeit im frühen Christentum zu erhalten. Die Art und Weise, wie der hellenistische Jude Paulus und die mehrheitlich

75. Die Sprache der Gegenseitigkeit durchzieht 7,10-16.

76. Vgl. dazu U. Poplutz, Athlet des Evangeliums. Eine motivgeschichtliche Studie zur Wettkampfmetaphorik bei Paulus (HBS 43), Freiburg i. Br. 2004, 273-276.

77. Dass in den ersten Jahrhunderten des Christentums auch Frauen Zugang zu dieser männlichen Rolle hatten, ist eine Entwicklung, die über Paulus hinausgreift. Vgl. dazu I. Stahlmann, Der gefesselte Sexus. Weibliche Keuschheit und Askese im Westen des Römischen Reiches, Berlin 1997. 
nicht-jüdischen Christen in Korinth in Konflikt geraten, zeigt, dass Männlichkeit von Anfang an implizit verhandelt wurde. Dabei stechen die folgenden Punkte hervor:

1. Paulus stellt die aktive Rolle des Mannes als Agens nicht grundsätzlich in Frage. Er macht jedoch mindestens zwei Qualifizierungen: Der christliche Mann gehört als Körper-Person zu Christus und er ist dazu verpflichtet, aus Liebe zu seinen Mitbrüdern und -schwestern wichtige Aspekte seiner männlichen Autonomie aufzugeben.

2. Paulus hat keine Sympathien für unmännliche Männer. Symbolische Genderüberschreitungen - wie etwa Männer mit langem Haar - gehören für ihn nicht in den öffentlichen Raum des christlichen Gottesdienstes.

3. Als öffentlicher Redner musste sich Paulus einer externen Evaluation seiner Männlichkeit aussetzen. Scheinbar erfüllte er nicht die physiognomischen Erwartungen in gehobenen römischen Zirkeln. ${ }^{78}$ Die kreuzestheologische Einsicht, dass Gottes Macht durch Schwäche wirkt, setzt einen kritischen Akzent gegenüber der Selbstrepräsentation, die die rhetorische Praxis seiner Zeit prägt.

4. Als Apostel vertritt Paulus eine Reihe von christologisch begründeten Werten, wie etwa Sanftmut ${ }^{79}$ oder die aus römischer Sicht sklavische Demut oder Niedrigkeit $^{80}$. Diese sind mit hegemonialen Konstruktionen von Männlichkeit kaum in Übereinstimmung zu bringen. ${ }^{81}$ Auf der anderen Seite findet sich die »Tapferkeit «

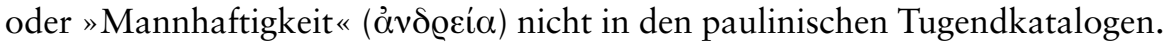

5. Paulus beschränkt Sexualverkehr auf die Ehe. Als ein »kleineres Übel « ist sie der legitime Ort zur Kontrolle sexueller Begierden. Sexualität bedeutet aber einen Entzug der kompletten Herrschaft Christi über den männlichen Körper und sie impliziert ein Eingeständnis fehlender Selbstbeherrschung. Für den Apostel ist die höchste Form männlicher Kontrolle daher die Selbstkontrolle.

78. Worin die Mängel konkret bestanden haben können, lässt sich nicht sagen. Bei der starken Normierung des männlichen Körpers würden eine dünne Stimme, X-Beine oder ein fleischiger Nacken bereits ein Problem darstellen.

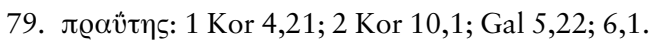

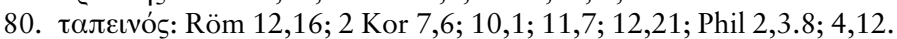

81. Natürlich stellt das Kerygma eines gekreuzigten Messias einen Affront gegenüber römischen Männlichkeitskonstrukten dar. 